\section{Aggression and multiple sclerosis}

SIR: Psychiatric symptoms, both cognitive and affective, have been reported frequently in multiple sclerosis (MS) (Grant, 1986). Euphoria and depression are the affective disorders most frequently encountered. No mention has been made of aggression. We present a young patient, who developed changes in personality, and aggressive behaviour, in conjunction with the acute onset of MS.

Case report. A previously healthy 17 -year-old man was admitted to hospital with acute loss of vision of the right eye, speech and balance disturbance, and an almost complete quadraparesis. A diagnosis of laboratory-supported definite MS (Poser, 1983) was established. Magnetic resonance imaging (MRI), using a mildly $\mathrm{T} 2$-weighted spin echo technique, demonstrated extensive periventricular white matter hyperintensities. Lesions were also found in the globus pallidus, the mesencephalon, the cerebellum, and bilaterally in the medial part of the temporal lobes. Subsequent high dose intravenous methylprednisolone treatment was followed by marked improvement of all but the visual symptoms. Soon afterwards he was able to resume his work.

After discharge, two months later, his family, employer, and general practitioner noticed progressive behavioural changes with an aggressive character, although his premorbid personality had been pleasant and co-operative. His aggressive behaviour led to provocations and fighting, followed by police contact on three occasions. One year after the initial symptoms, imprisonment followed extortion of an 83-year-old man. During this period he had an attack of optic neuritis of the left eye. At the time of imprisonment there were bilateral visual, and discrete cerebellar and sensory, signs. Psychiatric examination only revealed cognitive disturbances, such as slight memory loss, perseverance, and judgemental disturbances. The patient complained about a loss of sexual interest and impotence. There were no signs of depression or euphoria. Personal history revealed repeated assaults by his first stepfather. During four months imprisonment he stabilised, and showed no further aggressive behaviour. A second MRI showed a change in the lesions aforementioned. In the temporal lobes the old lesions had faded, but new lesions had appeared on both sides.

According to McGuire \& Troisi's(1989) definition, aggression is regarded as behaviour to cause physical injury to others, extended with verbal threats of physical violence. We report a previously healthy patient, with an acute and dramatic onset of MS associated with personality changes and aggressive behaviour. Aggression in this patient might have been caused by either psychogenic or organic factors, or both. Diminished cognitive control might have released emotions with aggressive connotations in relation to impotent anger caused by the MS. Unresolved enmity originating from the physical violence of his first stepfather could have added to an inner conflict.

Recently there has been increasing interest in the correlation of MS lesions, as seen on MRI, and neuropsychological impairment. In a study by Rao (1989) a strong correlation of the total lesion load with neuropsychological testing was found. A correlation of psychiatric disorders with MRI lesions in the temporal lobes has been reported by Honer (1987). Our patient showed bilateral MRI lesions in the medial part of his temporal lobes. The medial part of the temporal lobe, more specifically the hippocampus, corpus amygdaloideum and the gyrus parahippocampalis, contribute to the so-called 'limbic system'. This system is involved in memory, sexuality, and affective behaviour.

In conclusion, cognitive disturbances temporarily incapacitated impulse control and aggression regulation in a young man suffering from MS. The presence of organic lesions in the limbic system, and latent psychogenic factors, were also thought to have played a role in the development of his aggressive behaviour.

Grant, I. (1986) Neuropsychological and psychiatric disturbances in multiple sclerosis. In Multiple Sclerosis (eds W. I. McDonald \& D. H. Silberberg), pp. 134-152. London: Butterworths.

Honer, W. G., Hurwitz, T., LI, D. K. B., et al (1987) Temporal lobe involvement in multiple sclerosis patients with psychiatric disorders. Archives of Neurology, 44, 187-190.

MCGuire, M. T. \& Troist, A. (1989) Aggression. In Textbook of Psychiatry (eds H. I. Kaplan \& B. J. Sadock), pp. 271-283. Baltimore: Williams and Wilkins.

Poser, C. M., PAty, D. W., Scheinbeeg, L., et al (1983) New diagnostic criteria for multiple sclerosis: guidelines for research protocols. Annals of Neurology, 13, 227-231.

RAO, S. M., LEO, G. J., HAUGHTON, V. M., et al (1989) Correlation of magnetic resonance imaging with neuropsychological testing in multiple sclerosis. Neurology, 39, 161-166.

Psychiatric Department

L. KITS VAN WAVEREN

Department of Justice

Amsterdam

Department of Diagnostic Radiology

F. BARKHOF

Free University Hospital

P.O Box 7057

1007 MB Amsterdam

The Netherlands

Department of Neurology

Free University Hospital

Amsterdam

\section{Sudden cardiac death and antidepressants}

SIR: Antidepressant use has been associated with cardiac arrhythmias and sudden unexplained deaths, 
but this has not previously been reported in healthy young individuals. We report a case of sudden death during exercise in a fit 39-year-old male while undergoing antidepressant treatment.

Case report. The description of the mode of death of a fit 39-year-old male while out jogging was suggestive of acute cardiac failure. Post-mortem examination revealed no evidence of atherosclerosis or other conditions which together account for $94 \%$ of autopsy-proven causes of sudden natural death (Davies, 1989), and levels of medication were within therapeutic limits. There were no ECG abnormalities and, apart from a high degree of subjective distress, there were no risk factors for, or signs and symptoms of, ischaemic heart disease before commencing treatment with lofepramine ( $140 \mathrm{mg}$ daily) and temazepam (10 mg nocte PRN) for a major depressive disorder (DSMIII-R). The patient had reported troublesome anticholinergic side effects and an episode of palpitations - unrelated to exercise - in his fourth week of treatment, following an increase in the dose of lofepramine to $210 \mathrm{mg}$ daily, but physical examination 24 hours before his death revealed no abnormalities. A fatal arrhythmia (ventricular tachycardia which rapidly degenerated to ventricular fibrillation) was assumed. In the majority of cases, fatal arrhythmias are associated with myocardial infarction, and it was not possible to exclude an acute infarct, since post-mortem evidence may be lacking when death occurs rapidly. Lofepramine has not been associated with deaths in overdose (e.g. Cassidy \& Henry, 1987) but has been associated with cardiac arrhythmias and sudden unexplained deaths in the United Kingdom (but not in healthy young individuals). Between 1982 and 1990 there were 23 reports of tachycardia, 14 of cardiac arrhythmia (2 deaths), two of non-fatal cardiac arrest, and four sudden deaths. On the basis of equivocal evidence, an open verdict was reached at inquest.

It is possible that antidepressants with anticholinergic side effects may have an indirect role in the evolution of sudden cardiac death (SCD) by depressing baroreflex sensitivity (BRS). The baroreflex results in a slowing of the heart rate in response to a rise in systolic blood pressure, and is therefore protective against the progression of tachyarrhythmias. It is known that the risk of SCD is greatly increased in patients with autonomic neuropathies affecting the vagal nerve, and it has been established, in canine models and post-infarction patients, that a depressed BRS (primarily reflecting an impairment in the vagal efferent component of the baroreflex) is associated with a greater susceptibility to ventricular fibrillation and subsequent SCD (e.g. Cripps \& Camm, 1989).

Cassidy, S. \& Henry, J. (1987) Fatal toxicity of antidepressant drugs in overdosage. British Medical Journal, 295, 1021-1024.

Da vies, M. J. (1989) In Current Approaches: Sudden Cardiac Death (eds A. J. Camm, J. C. Malkin \& M. L. Page). Southampton: Duphar Medical Relations.
CRIPPS, T. R. \& CAMM, A. J. (1989) Prediction of arythmic events in patients following myocardial infarction. Clinical Cardiology, 12, $661-665$.

University Department of Psychiatry

ToNI Lock

Royal Liverpool Hospital

Box 147

Liverpool L69 3BX

Broadgreen Hospital

Liverpool

University Department of Psychiatry

Royal Liverpool Hospital

William Kenyon

M. T. Abou-SAleh

\section{Hair loss associated with fluoxetine}

SIR: I would like to report a case of hair loss in a patient taking fluoxetine, which is rare.

Case report. Mrs A, a 72-year-old, married white woman with a one-year history of depression, met DSM-III-R criteria for major depression without psychotic features. Her symptoms included psychomotor retardation, slow low volume speech, poor energy level and sleep disturbance, but no suicidal ideation. She had no history before this event of any psychiatric disorder, including alcohol or drug abuse. Her laboratory tests, which included a total with differential blood count, liver function tests, electrolytes, thyroid function studies (T3, T4 and TSH), venereal disease research laboratory test (VDRL), and urine analysis, were within normal limits. She had received an adequate trial of nortriptyline and trazodone without success. A trial of fluoxetine ( $20 \mathrm{mg} /$ day) was begun with her Hamilton Rating Scale for Depression (HRSD) score being 24 on a 21 -item scale. She began noticing significant hair loss from the scalp within two weeks without any other dermatological problem. The fluoxetine was immediately discontinued, but she continued to lose scalp and body hair. A complete medical evaluation revealed no other cause for the hair loss. She was not taking other medications concomitantly, nor did she suffer from any significant ongoing medical problems. Her depression was treated successfully with electroconvulsive therapy, the HRSD score reducing to four. The hair loss continued, and at 18-month follow-up she remains without any scalp or body hair, but is doing well as far as depression is concerned.

We are aware of only one other case of severe hair loss with fluoxetine (Jenicke, 1991). As fluoxetine is a widely used antidepressant, these reports suggest that there might be a wide spectrum of hair loss, from obvious cases which come to medical attention to subtle ones which clinicians should look for. 